MODELING, IDENTIFICATION AND CONTROL, 1992, VOL. 13, NO. 2, 113-125

doi:10.4173/mic.1992.23

\title{
Variable selection for decentralized control
}

\author{
SIGURD SKOGESTAD $†$ and MANFRED MORARI $\ddagger$
}

Keywords: DIC, relative gain array $(R G A)$, steady-state, integral control, interactions

Decentralized controllers (single-loop controllers applied to multivariable plants) are often preferred in practice because they are robust and relatively simple to understand and to change. The design of such a control system starts with pairing inputs (manipulated variables) and outputs (controlled variables). For a $\mathrm{n} \times \mathrm{n}$ plant there are $n$ ! possible pairings, and there is a great need for screening techniques to quickly eliminate undesirable pairings. In this paper we present several tests for eliminating pairings which are not decentralized integral controllable (DIC). A system is DIC if there exists a stabilizing decentralized controller with integral action such that the gains of the individual loops may be reduced independently without introducing instability. Note that DIC is a property of the plant and the chosen pairings. The tests presented are in terms of different measures of the sign of steady state gain matrix; including the RGA, the determinant and eigenvalues. The relationship to previously presented results is discussed in detail.

\section{Introduction}

Decentralized control implies the use of single-loop controllers to control multivariable processes. This means that for any particular choice of pairing of controlled and manipulated variables we can rearrange the plant $G$ such that the controller $C$ is diagonal

$$
C=\operatorname{diag}\left\{c_{i}\right\}
$$

The constraints on the controller structure invariably lead to performance deterioration when compared to the case with a full controller matrix. Still, decentralized controllers are very common in practice, for the following reasons:

(i) ease of implementation,

(ii) simplified design,

(iii) decentralized turning,

(iv) robustness with respect to model error, and

(v) ease of making system failure tolerant.

In short, single loop controllers are preferred by the operators because they are robust and relatively simple to understand and to change.

The designer of decentralized controllers is faced with the issues of (1) pairing outputs and inputs, and (2) controller design (tuning) of the individual loops. This paper addresses the pairing problem. Even for relatively small plants, there are many

Received 6 March 1992.

†Chemical Engineering, Norwegian Institute of Technology (NTH), N-7034 Trondheim, Norway.

† Chemical Engineering, 206-41, California Institute of Technology, Pasadena, CA 91125, U.S.A.

This paper was presented at the American Institute of Chemical Engineers Annual Meeting, Washington DC, Nov. 1988, Paper $126 f$. 
decentralized control systems to choose from. Consider pairing of single loops. Then for a $2 \times 2$ plant there are two alternatives, a $3 \times 3$ plant offers 6 , a $4 \times 4$ plant 24 , a $5 \times 5$ plant 120 , etc. Thus efficient screening techniques are needed which are capable of eliminating quickly inappropriate control structures.

In this paper the criterium chosen is that the controller structure should be 'Decentralized Integral Controllable' (DIC). A plant $G$ (corresponding to a particular choice of pairings) is DIC if there exists a stabilizing decentralized controller with integral action (no offset) such that the gains of the individual loops can be reduced independently without introducing instability. In particular, DIC implies that any subset of loops can be detuned or taken out of service (put in 'manual') without affecting stability. Note that DIC is a property of the plant and the particular control structure (pairing) chosen.

Necessary conditions for DIC are of particular interest, since a violation of such a condition means that DIC is not possible and the corresponding pairing may be eliminated. For most plants the majority of the alternatives may be eliminated using such conditions. To select the best of the few remaining alternatives, sufficient conditions for DIC are more useful. In this paper several necessary conditions in terms of the steady state gain matrix are presented. Some of the results have been presented elsewhere, but their interpretation in terms of DIC is new. It is stressed that only steady state data are needed.

The main reason for the problems encountered with decentralized controllers are the 'interactions' caused by the off-diagonal elements in the plant $G$. If these elements are 'small' then interactions are weak and decentralized control is simple. If the interactions are large, then it might happen that the sign of the plant gain between a specific plant input and output changes sign as other loops are closed. Integral control, which is known to depend on knowing the plant gain, is then not possible. All of the conditions presented are therefore in terms of avoiding pairings where the plant gain may change sign as other loops are changed.

A good discussion of the importance of the pairing problem is presented by Nett and Spang (1987). Bristol (1966) introduced the relative gain array (RGA) as a criterion for choosing the best variable pairing, and this measure continues to be the one most often used. Niederlinski (1971) proposed considering the sign of the determinant of the plant as a screening tool. McAvoy (1983) and Grosdidier et al. (1985) discuss the use of the RGA in more detail and provide theoretical justification for Bristol's rule of avoiding pairings corresponding to negative relative gains. Grosdidier et al. (1985) also present several conditions for a plant to be integral stabilizable (IS) or integral controllable (IC), which upon reformulation turn out to be useful tools for eliminating pairings. Mijares et al. (1986) introduced the 'Jacobi Eigenvalue criterion' as a tool for selecting the best pairing. This criterion is closely related to the SSV $(\mu)$-interaction measure introduced by Grosdidier and Morari (1986). Yu and Luyben (1986) present three rules for eliminating unworkable pairings. The first is based on the RGA, the second on Niederlinski's result, and the third involving MIC is based on Theorem 7 (determinant condition for IS) in Grosdidier et al.(1985). Grosdidier and Morari (1987) introduced the property of block-IC. This property is easier to satisfy than DIC, since DIC implies block-IC, but not conversely.

The objective of this paper is to show that all the above-mentioned conditions are related rigorously to DIC and IC, and to derive some new conditions for DIC.

Throughout the paper we assume that the plant $G(s)$ is a square, open-loop stable, strictly proper transfer matrix. The steady state value of this matrix is $G(0)$. A general 


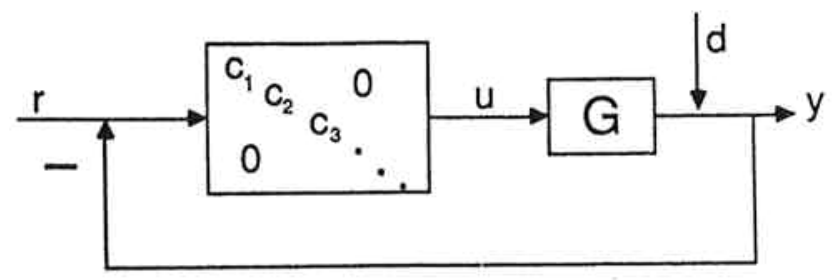

Figure 1. Decentralized control structure.

decentralized control system is shown in Fig. 1. The notation is summarized at the end of the paper. All rules and theorems are given for the case of single loop pairings, but most of them are easily extended to blocks (see Manousiouthakis et al. 1986, and Grosdidier and Morari 1987).

\section{Summary of rules for eliminating undesirable pairings}

Below we present a summary of rules for pairing selection. The rules are subsequently proved in $\S 4$.

Yu and Luyben (1986) present three rules for eliminating what they call unworkable variable pairings:

Rule 1. Eliminate pairings with negative RGA's.

Rule 2. Eliminate pairings with negative Niederlinski Indexes

$$
\mathrm{NI}=\frac{\operatorname{det}(G(0))}{\prod_{i=1}^{n} g_{i i}}
$$

Rule 3. Eliminate pairings with negative Morari Indexes of Integral Controllability, $\mathrm{MIC}=\operatorname{Re}\left\{\lambda\left(G^{+}(0)\right)\right\}$.

In fact, we will show that violation of any of these three rules imply that the plant is not decentralized integral controllable (DIC) with this choice of variable pairings. With respect to rule 3, Yu and Luyben claim that a negative MIC-value 'shows that the variable pairing will produce an unstable closed-loop system'. This is not necessarily correct (see Example 2 below), but it might happen if one of the loops is detuned since the system is not DIC. In $\S 4$ we establish rule 3 rigorously in terms of DIC. We also show that rule 2 (involving NI) is redundant, because rule 3 always implies rule 2 as special case.

Furthermore, the following new rules for eliminating pairings for which DIC is not possible are established:

Rule 4. Eliminate pairings with $\operatorname{Re}\{\lambda(E(0))\}<-1 ; E=\left(G-G_{\text {diag }}\right) G_{\text {diag. }}^{-1}$.

Rule 5. Eliminate pairings for which there exists a $K$ (diagonal matrix with positive entries) which yields $\operatorname{Re}\left\{\lambda\left(G^{+}(0) K\right)\right\}<0$.

Rules 3 and 4 are special cases of rule 5 . They are derived from rule 5 by choosing $K$ equal to $I$ (the identity matrix) and $G_{\text {diag }}^{+}(0)^{-1}$, respectively. Rule 4 involves the matrix $E$ used in the interaction measures derived by Grosdidier and Morari (1986). Rule 4 is equivalent to eliminating pairings with eigenvalues of the 'Jacobi iteration matrix' greater than one (Mijares et al., 1986). Numerical evidence proves that none of rules 1,3 or 4 is mutually redundant, and all three are therefore useful. 
One important advantage with the RGA (rule 1) is that it is very simple to compute and does not have to be recomputed to investigate alternative pairings. This follows since a permutation of the rows or columns in the plant $G$, corresponding to a change in pairings, results in the same permutation in the RGA (Bristol 1966). Consequently, one should always start by eliminating undesirable pairings according to rule 1 (RGA), and subsequently use rules 3 and 4 to screen the remaining alternatives.

Rules 1-5 above may all be reformulated as necessary conditions for DIC. This means that a plant that does not pass these tests is not DIC, but there may be other plants that pass the tests, but still turn out not to be DIC.

There also exist sufficient conditions for DIC. One of these is in terms of the structured singular value $\mu$ (Doyle 1982) of $E$, and yields the rule:

Rule 6. Prefer pairings with $\mu(E(0))<1$.

(here $\mu$ is computed with respect to the structure of $C$ ). Note that

$$
\rho(E) \leqslant \mu(E) \leqslant \rho(|E|)
$$

and we therefore have that the eigenvalues of $E(0)$ should always be greater than -1 (rule 4), and their magnitude $\rho(E(0))$ preferably less than 1 (rule 6 and Eqn. (1)). The criterion that the spectral radius of $E(0), \rho(E(0))$, should be less than one is equivalent to the 'Jacobi Eigenvalue Condition' of Mijares et al. (1986). This condition is rigorously related to IC (see below), but not to DIC. For DIC, $\mu(E(0))$ is the right measure.

There exists no simple necessary and sufficient condition for DIC. If it is not possible to find any $K$ which satisfies the criterion for elimination in rule 5, then, under some minor technical conditions, we may conclude that the system is DIC. That is, DIC is equivalent to

$$
\min _{K} \min _{i} \operatorname{Re}\left\{\lambda_{i}\left(G^{+}(0) K\right)\right\}>0
$$

However, this last condition is difficult to test, and therefore of limited practical value.

\section{Definitions}

Decentralized Integral Controllability. A plant $G$ (corresponding to a particular pairing) is decentralized integral controllable (DIC) if it is possible to design a diagonal controller for this plant which (1) has integral action (no offset for tracking), (2) yields stable individual loops, (3) is such that the system remains stable when all loops are closed simultaneously and (4) has the property that each loop gain may be reduced independently with a factor $\epsilon_{i}\left(0 \leqslant \epsilon_{i} \leqslant 1\right)$ without introducing instability.

Decentralized controllers are frequently used in process control, and it is obviously desirable that they satisfy the above requirements for DIC. Note that the property of DIC depends on the particular pairings chosen: the plant may satisfy DIC for one choice of pairings, but not for another. To satisfy condition (2) the controller must be such that the individual loop gains $g_{i i} c_{i}$ are all positive. Also note that property (4) implies (2) since one particular choice of loop gains is to have all but one loop with zero. gain.

The definition of DIC is similar to that of 'Integral Controllability' (IC) introduced by Grosdidier et al. (1985).

Integral Controllability. A system (plant and controller) is integral controllable (IC) if (1) the controller has integral action, (2) the overall system is stable, and (3) all controller gains may be reduced by the same factor $\epsilon(0<\epsilon<1)$ without introducing instability. 
Note from the definitions that for IC all the gains are reduced by the same amount, while for DIC each loop gain may be reduced at a different rate. Consequently, there may be a particular decentralized controller which satisfies IC, but this does not necessarily imply that the plant with this choice of pairings satisfies DIC. However, the reverse holds:

$$
\mathrm{DIC} \Rightarrow \mathrm{IC} \text { (with any decentralized controller with positive loop gains) }
$$

Thus control structures which fail the IC test can be eliminated when searching for a plant which is DIC. Another somewhat subtle point is that whereas IC is a property that depends on both the plant $G$ and the controller $C$, the property of DIC depends only on the plant. This follows because we are allowing each loop gain to be reduced by an arbitrary amount which is equivalent to allowing any ratio between the elements in the controller and we are therefore considering all possible diagonal controllers (at least at steady state). From this point of view DIC is a much more useful property than IC since it is an inherent plant property independent of the particular choice of controller.

\section{Theorems}

\subsection{Necessary conditions for DIC}

The basis for all the results presented below is that negative feedback is needed to have stability under integral control, that is, we must know the sign of the plant gain. We will see that all of the results involve different expressions for the plant gains, either in terms of the determinant, eigenvalues or relative gains. We will first recall three results for DIC and IC given by Grosdidier et al.(1985) - though they are not explicitly written in this form.

Theorem 1 (basis for rule 1). Assume $C(s)$ is a diagonal controller and that $G(s) C(S)$ is proper (always satisfied for any real system). Then

$$
\mathrm{RGA}_{i i}(G(0))<0 \text { for some } i \Rightarrow \text { not DIC }
$$

or equivalently (see Appendix)

$$
\mathrm{DIC} \Rightarrow \mathrm{RGA}_{i i}(G(0)) \geqslant 0, \quad \forall i
$$

Here $\operatorname{RGA}_{i i}(G)$ denotes the $i$ 'th diagonal element of the RGA of $G$.

Proof. Follows from Theorem 6 in Grosdidier et al. (1985).

The rule of avoiding pairings corresponding to negative RGA-elements goes back to Bristol (1966), but it was proved rigorously only recently. Note that $i j$ 'th element of the RGA is defined as

$$
\mathrm{RGA}_{i j}=\frac{\left(\partial y_{i} / \partial u_{j}\right)_{u_{k, k \neq j}}}{\left(\partial y_{i} / \partial u_{j}\right)_{y_{l, l \neq i}}}=\frac{g_{O L}}{g_{C L}}
$$

that is, it represents the ratio of the gain from $u_{j}$ to $y_{i}$ in open loop (other $u$ 's constant) and closed-loop (other $y$ 's constant). If the sign of this gain changes as we change or close other loops, then we are not able to apply negative feedback in all cases, and the plant is not DIC. 
Theorem 2 (basis for rule 2). Assume $C(s)$ is a diagonal controller, $G(s)$ is stable and that $G(s) C(s)$ is strictly proper (always satisfied for any real system). Then

or equivalently

$$
\frac{\operatorname{det}(G(0))}{\prod_{i=n}^{n} g_{i i}}<0 \Rightarrow \text { not DIC }
$$

$$
\operatorname{det}\left(G^{+}(0)\right)<0 \Rightarrow \text { not DIC }
$$

Proof. Follows from Theorem 3 in Grosdidier et al. (1985).

$(4 a)$ is Niederlinski's result which tells us that we should avoid using decentralized control on pairings which have the sign of the plant (given in terms of its determinant) different from the product of the plant gains for the loops. Again, this is a condition for avoiding the use of positive feedback.

Most of the new results in this paper (Theorems 4, 5 and 6) are based on the following theorem in terms of IC:

Theorem 3 (eigenvalue condition for IC). Write the controller $C(s)$ with integral action as $C(s)=\frac{k}{s} \hat{C}(s)$. Then there exists a $k^{*}>0$ such that the system is stable for all $0 \leqslant k \leqslant k^{*}$ (i.e., the system is IC) if

$$
\operatorname{Re}\left\{\lambda_{i}(G \hat{C}(0))\right\}>0, \quad \forall i
$$

and there does not exist such a $k^{*}$ (i.e., the system is not IC) if

$$
\operatorname{Re}\left\{\lambda_{i}(G \hat{C}(0))\right\}<0, \quad \forall i
$$

Proof. See Theorem 7 in Grosdidier et al. (1985). The proof is based on MacFarlanes generalized Nyquist theorem in terms of the characteristic loci.

In words, the real part $(\mathrm{Re})$ of all the eigenvalues of $G \hat{C}(0)$ must be positive to have IC, i.e., the eigenvalues must all be in the right half plane. Furthermore, if we disregard the few cases where the eigenvalues of $G \hat{C}(0)$ are one the $j \omega$-axis (purely complex), this is a necessary and sufficient condition. The following condition in terms of DIC when $C$ is diagonal is easily derived from $(5 b)$ :

Theorem 4 (basis for rule 5). Let $K$ be a diagonal matrix with real, positive (nonzero) entries. Then

$$
\min _{i} \operatorname{Re}\left\{\lambda_{i}\left(G^{+}(0) K\right)<0, \text { for some } K \Rightarrow\right. \text { not DIC }
$$

Proof. Consider a specific diagonal controller $C$ which yields positive individual loop gains $g_{i i} c_{i}$ (needed to satisfy property 2 in the definition of DIC). Write $G \hat{C}(0)=G^{+}(0) K$ where $K=|\hat{C}(0)|$ has only positive elements. Then from Theorem 3, Eqn. $(5 b)$ : $\operatorname{Re}\left\{\lambda\left(G(0)^{+} K\right)\right\}<0 \Rightarrow$ not IC for this diagonal controller $\Rightarrow$ not DIC (the last implication follows from Eqn. 2).

Theorem 4 by itself is not too useful because it requires specifying a controller. However, the following two results are obtained by choosing the diagonal controller gains $K$ as $I$ and $G_{\text {diag }}^{+}(0)^{-1}$, respectively.

Theorem 5 (basis for rule 3).

$$
\min _{i} \operatorname{Re}\left\{\lambda_{i}\left(G^{+}(0)\right)\right\}<0 \Rightarrow \operatorname{not} \mathrm{DIC}
$$

Theorem 6 (basis for rule 4).

$$
\min _{i} \operatorname{Re}\left\{\lambda_{i}\left(G(0) G_{\mathrm{diag}}^{-1}(0)\right)\right\}<0 \Rightarrow \text { not DIC }
$$


Theorem 5 is the basis for the MIC-rule (rule 3) which has been presented previously by $\mathrm{Yu}$ and Luyben (1986), but without any proof. Furthermore, Yu and Luyben interpret the MICs in terms of IC and not in terms of DIC as they should.

Note that $\operatorname{det}\left(G^{+}\right)$which appears in Theorem 3 is the product of the eigenvalues of $G^{+}$(MICs) which appear in Theorem 5. An even number of negative eigenvalues of $G^{+}$ will result in a positive $\operatorname{det} G^{+}$, but the reverse is not possible (i.e., negative $\operatorname{det} G^{+}$ cannot yield all positive eigenvalues; this follows since any complex eigenvalues come in pairs). Consequently, Theorem 4 yields 2 as a special case (but not vice versa), and the NI therefore contains less information than the MICs. Use of rule 3 therefore makes rule 2 redundant.

Rule 4 follows from Theorem 6 since $\lambda(E(0))=\lambda\left(G(0) G_{\text {diag }}^{-1}(0)\right)-1$. A similar result to Theorem 6, but in terms of $\operatorname{Re}\{\lambda(E(0))\}>-1$ as a necessary and sufficient condition for IC (our result is that it is a necessary condition for DIC), has been derived by Mijares et al. (1986) (Eqn. 37 in their paper). They consider the eigenvalues of the 'Jacobi Iteration Matrix' $A=I-G_{\text {diag, }}^{-1}$, but this is essentially the same matrix as $E$ since $\lambda_{i}(E)=$ $-\lambda_{i}(A)$. For an alternative proof of Mijares's result see Skogestad and Morari (1987).

Other theorems similar to Theorems 5 and 6 can be derived by making other more or less arbitrary choices for the matrix $K$ : If we can show for a particular diagonal controller that IC is not possible, then we know that DIC is not possible for this plant. However, the two choices made above seen to be the most reasonable, and also tie in very nicely with results presented previously.

\subsection{Sufficient conditions for DIC}

$\mu$-conditions. The matrix $E=\left(G-G_{\text {diag }}\right) G_{\text {diag }}^{-1}$ in rule 4 appears in the interaction measures introduced by Grosdidier and Morari (1986). From Corollary 2.1 in their paper we derive that a sufficient condition for having IC is that $\rho(E(0))$ (magnitude of largest eigenvalue) is less than one, or equivalently

$$
\rho(E(0))<1 \Rightarrow \mathrm{IC}
$$

Note that this does not guarantee DIC since one requirement of using $\rho(E)$ is that all loops $g_{i i} c_{i}$ are identical (i.e., $\hat{C}=G_{\text {diag }}^{-1}$ ) and the loops cannot be detuned independently. However, an equivalent condition in terms of DIC results if $\rho(E)$ is replaced by $\mu(E)$, that is (Theorem 7 in Grosdidier and Morari 1986)

$$
\mu(E(0))<1 \Rightarrow \mathrm{DIC}
$$

The generalization to DIC follows since the use of $\mu(E)$ allows the individual loops to be different. $\mu(E(0))$ can be used to tell that DIC is satisfied for a particular pairing. However, it cannot be used to eliminate variable pairings since it may be possible to achieve DIC for a plant even though $\rho(E(0))$ and thereby also $\mu(E(0))$ (recall Eqn. 1$)$ is greater than 1 . This is illustrated in the discussion on $2 \times 2$ plants below.

However, the main advantage with $\mu(E(0))<1$ is that interactions are small and the controllers for each loop may easily be designed independently (that is, on the basis of $G_{\text {diag }}$ only) (see Grosdidier and Morari 1986, and Skogestad and Morari 1989, who provide guidelines for the design). Consequently, we prefer pairings with $\mu(E)$ (and $\rho(E)$ ) less than 1 because we (i) are guaranteed DIC and IC, and (ii) may easily design the loops independently. This is the basis for rule 6.

Block-IC. The definition of DIC is similar to that of block-IC (here denoted loop-IC since we only consider single loops and not blocks) introduced by Grosdidier and 
Morari (1987). Loop-IC implies that one loop at the time may be detuned, but DIC is stricter since it all allows all loops to be detuned simultaneously in an arbitrary fashion. Theorem 6 in Grosdidier and Morari (1987) says that, provided the system is IC with a diagonal controller in the first place, loop-IC of all loops is guaranteed if and only if the RGA has positive diagonal elements. (Note that a separate test is needed in addition to test IC.) The only if part is not too surprising from the DIC-condition in Theorem 1. The main new information in this result is therefore

$$
\operatorname{RGA}(G(0))_{i i}>0, \quad \forall i \Rightarrow \text { loop- }-\mathrm{IC}
$$

That is, positive RGA-elements guarantee that loops may be detuned one at a time (provided the system is IC in the first place). We have another result (Eqn. 10) which also guarantees the same

$$
\mu(E(0))<1 \Rightarrow \text { DIC } \Rightarrow \text { loop }- \text { IC }
$$

However, (11), which is necessary and sufficient, is of course more useful (less conservative) than (12).

\subsection{Necessary and sufficient conditions for DIC}

Theorem 7. Let $K$ be any diagonal matrix with real, positive (nonzero) entries. Let

$$
\Omega(G(0)) \triangleq \min _{K} \min _{i} \operatorname{Re}\left\{\lambda_{i}\left(G^{+}(0) K\right)\right\}
$$

Disregard the case when $\Omega$ of $G(0)$ or any of its subsystems (obtained by choosing some $c_{i}=0$ ) is exactly zero (e.g., caused by $G(0)$ or any of its subsystems being singular). Then Theorem 3 is necessary and sufficient for DIC, that is

$$
\Omega(G(0))>0 \Leftrightarrow \mathrm{DIC}
$$

Proof. This result follows from the definition of DIC and Theorem 3: From Theorem 3 we know that conditions 1 to 3 in the definition of DIC will be satisfied if

$$
\left.\operatorname{Re}\left\{\lambda\left(G^{+}(0) K\right)\right)\right\}>0
$$

Condition 4 with $0<\epsilon_{i}<1$ is satisfied if Eqn. (6) is satisfied for all possible $K$ 's (follows from Theorem 3, $(5 a)$ by considering all possible diagonal controllers, that is, each gain may be reduced independently): Condition 4 with some $\epsilon_{i}=0$ is not covered by this test (Eqn. 13). This case corresponds to deleting rows and corresponding columns in $G(0)$, and considering instability of the remaining subsystem under decentralized control. Simple limiting arguments show that stability of these subsystems is also guaranteed by Eqn. 15 provided neither of the submatrices yield $\Omega$ exactly equal to zero (e.g., caused by singular submatrices). For example, this means that pairing on elements with zero gain is disregarded. Summing up we have under this condition that

$$
\left.\min _{K} \min _{i} \operatorname{Re}\left\{\lambda_{i}\left(G^{+}(0) K\right)\right)\right\}>0 \Rightarrow \text { DIC }
$$

Combining this with (6) and assuming in addition that $\Omega(G(0))$ is not exactly zero (e.g., caused by $G(0)$ being singular) we arrive at condition (14).

As mentioned before condition (14) is of limited usefulness since it is difficult to test. In particular, if the plant is DIC then $\Omega(G(0)) \rightarrow 0^{+}$(choose small elements in $K$ ), and if it is not DIC then $\Omega(G(0)) \rightarrow-\infty$ (choose large elements in $K$ ). In our numerical studies we have used a general purpose optimization routine which seems to have worked satisfactorily. The optimization is stopped as soon as a $K$ which yields negative eigenvalues of $G^{+}(0) K$ is found. 


\section{4. $2 \times 2$ Plants}

Theorem 8 . Consider $2 \times 2$ plants with $G(0)$ nonsingular and both diagonal elements nonzero. Then theorems $1-5$ (and rules 1-5) are all equivalent and are all necessary and sufficient for DIC

$$
\mathrm{DIC} \Leftrightarrow \mathrm{RGA}_{11}>0 \Leftrightarrow \mathrm{NI}>0 \Leftrightarrow \mathrm{MIC}>0 \Leftrightarrow \operatorname{Re}\{\lambda(E(0))\}>-1
$$

Proof. This follows from the following facts:

1. Eqn. 14 is necessary and sufficient for DIC provided $G(0)$ is nonsingular and the diagonal elements are both nonzero.

2. For $2 \times 2$ plants $G^{+}(0) K$ will have all its eigenvalues in the right half plane if and only if $G^{+}(0)$ has all its eigenvalues in the right half plane (this fact is easily established by applying the Routh test to the characteristic polynomial). Consequently, eqn. (14) and Theorems 3-5 are equivalent in this case.

3. For $2 \times 2$ plants $G^{+}(0)$ has all its eigenvalues in the right half plane if and only if $\operatorname{det}\left(G^{+}(0)\right)$ is positive. Consequently, Theorems 5 and 2 are equivalent.

4. For $2 \times 2$ plants both the diagonal elements of the RGA are equal and furthermore $\mathrm{RGA}_{11}=1 / \mathrm{NI}$. Consequently, Theorems 1 and 2 are equivalent.

$\mu$-conditions. For $2 \times 2$ plants $\rho(E)=\mu(E)$ and condition (12) becomes (Grosdidier and Morari 1986)

$$
\rho(E(0))<1 \Rightarrow \mathrm{DIC}
$$

Furthermore, (18) is equivalent to (Grosdidier and Morari 1986)

$$
\operatorname{RGA}_{11}(G(0))>0.5 \Rightarrow \mathrm{DIC}
$$

However, from Theorem 8 we have another necessary and sufficient condition for DIC

$$
\operatorname{RGA}_{11}(G(0))>0 \Leftrightarrow \mathrm{DIC}
$$

Consequently, whereas we know from (20) that it is possible to design a controller which is DIC for $2 \times 2$ plants with positive diagonal RGA-elements, condition (19) indicates that the RGA-elements should be greater than 0.5 . Conditions (18) and (19) are therefore conservative (sufficient only). This does not mean that there might not be factors other than DIC that may favour choosing pairings with RGA-elements larger than 0.5 . For example, closed-loop performance may be better because of less interactions which may make it possible to use a higher gain. (The definition of DIC just says there will exist some detunable diagonal controller with integral action that yields stability; it does not guarantee good performance.)

\section{Examples}

Example 1.

$$
\begin{aligned}
G(0) & =\left(\begin{array}{rr}
1 & -2 \\
1 & 1
\end{array}\right) \\
\operatorname{RGA}(G(0)) & =\left(\begin{array}{ll}
0.33 & 0.67 \\
0.67 & 0.33
\end{array}\right) \\
\mathrm{NI}=3, \quad \mathrm{MIC} & =\lambda\left(G^{+}(0)\right)\{1 \pm 1.414 j\} \\
\lambda(E(0)) & =\{ \pm 1.414 j\}
\end{aligned}
$$


This is a $2 \times 2$ plant and rules $1-5$, which are necessary and sufficient for DIC in this case, all tell us that this plant is DIC. This is the case even though $\rho(E(0))=\mu(E(0))=1.41$ which means that sufficient condition (10) for DIC is not satisfied. Note that the plant is DIC also with the reverse pairing (diagonal RGAelements are 0.67), and in addition $\mu(E(0))=0.71<1$ in this case. The reverse pairing is therefore preferable according to rule 6.

Example 2.

$$
\begin{aligned}
G(0) & =\left(\begin{array}{crr}
10 & 0 & 20 \\
0.2 & 1 & -1 \\
11 & 12 & 10
\end{array}\right) \\
\operatorname{RGA}(0) & =\left(\begin{array}{ccc}
4.58 & 0 & -3.58 \\
1 & -2.5 & 2.5 \\
-4.58 & 3.5 & 2.08
\end{array}\right) \\
\mathrm{NI}=0.48, \quad \mathrm{MIC} & =\lambda\left(G^{+}(0)\right)=\{-3.00,-0.65,24 \cdot 7\} \\
\lambda(E(0)) & =\{-0.59 \pm 0.23 j, 1 \cdot 19\}
\end{aligned}
$$

Here $\lambda(E(0))$ and NI are inconclusive, the MIC- and RGA-values tell that the plant is not DIC with this pairing.

However, this does not necessarily mean the plant is not integral controllable (IC). Consider the following diagonal controller consisting of three SISO controllers; $C=\frac{k}{s} \hat{C}, \hat{C}=\operatorname{diag}\{0 \cdot 1,1,0 \cdot 1\}$. This controller yields stable individual loops since the loop gains are positive. Furthermore, the matrix $G \hat{C}(0)$ has all eigenvalues in the right half plane $(\lambda(G \hat{C}(0))=\{0.41 \pm 0.23 j, 2.19\})$, and we know from Theorem 3 that the controller yields a system which is integral controllable (IC). This means that the system will remain stable if all the gains in the controller are detuned by the same amount. However, if each loop is detuned in an arbitrary fashion, the system may become unstable. For example, we know from the negative MIC-values, that if we detune the controller gain in $\hat{C}$ for the second loop from 1 to $0 \cdot 1$, and keep the other controller gains fixed at $0 \cdot 1$, then the system will become unstable. This kind of conditional stability is clearly undesirable and this is the reason why one in practice prefers plants which are DIC and not only IC.

\section{Example 3.}

This model of a sidestream column is given by Elaahi and Luyben (1985)

$$
G(0)=\left(\begin{array}{rrrr}
8.72 & 2.81 & 2.98 & -15.80 \\
6.54 & -2.92 & 2.50 & -20.79 \\
-5.82 & 0.99 & -1.48 & -7.51 \\
-7.23 & 2.92 & 3.11 & 7.86
\end{array}\right)
$$




$$
\begin{aligned}
\operatorname{RGA}(G(0)) & =\left(\begin{array}{rlrr}
0.41 & 0.47 & -0.06 & 0.17 \\
-0.20 & 0.45 & 0.32 & 0.44 \\
0.40 & 0.08 & 0.17 & 0.35 \\
0.39 & 0.001 & 0.57 & 0.04
\end{array}\right) \\
\mathrm{NI} & =-18.65, \quad \mathrm{MIC}=\{-9.69,4.74,6.05,19.88\} \\
\lambda(E(0)) & =\{-3.25,1.88,0.69 \pm 0.162 j\}
\end{aligned}
$$

Here the RGA is inconclusive, the three other tests tell that the plant is not DIC with choice of pairings.

\section{Example 4.}

$$
\begin{aligned}
G(0) & =\left(\begin{array}{rrc}
0.5 & 0.5 & -0.004 \\
1 & 2 & -0.01 \\
-30 & -250 & 1
\end{array}\right) \\
\operatorname{RGA}(G(0)) & =\left(\begin{array}{rrr}
-1.56 & -2.19 & 4.75 \\
3.12 & 4.75 & -6.87 \\
-0.56 & -1.56 & 3.12
\end{array}\right) \\
\mathrm{NI} & =0.16, \quad \mathrm{MIC}=\{0.049 \pm 0.21 j, 3.40\} \\
\lambda(E(0)) & =\{-0.82 \pm 0.17 j, 1.64\}
\end{aligned}
$$

Here only the RGA allows us to conclude that this pairing is not DIC; all the other tests are inconclusive. In fact, from the RGA we see that it is impossible to rearrange the plant such that all diagonal RGA-elements are positive. Consequently, this plant is not DIC for any choice of pairings.

\section{Example 5.}

This example was first presented by Niederlinski (1971) and is also used by Mijares et al. (1986)

$$
\begin{aligned}
G(0) & =\left(\begin{array}{rrr}
1.0 & -0.1 & 1.0 \\
-0.5 & 0.6 & 0.1 \\
-0.2 & -0.8 & 0.3
\end{array}\right) \\
\mathrm{RGA} & =\left(\begin{array}{rrr}
0.34 & -0.02 & 0.68 \\
0.50 & 0.39 & 0.11 \\
0.16 & 0.63 & 0.21
\end{array}\right) \\
\mathrm{NI} & =4.26, \quad \mathrm{MIC}=\{0.27 \pm 0.70 j, 1.35\} \\
\lambda(E(0)) & =\{-0.52 \pm 1.36 j, 1.05\}
\end{aligned}
$$

This choice of pairings is not excluded by any of the pairing rules, and we may therefore expect it to be DIC. It is certainly IC, for example with a controller with $C(0)=k / s I$ (since the MIC's are positive). Furthermore, we know from Eqn. 11 that this plant is loop-IC, that is, we may detune one loop at the time in an arbitrary fashion. It then 
seems extremely likely that the plant is also DIC. Indeed, a numerical search gave $\Omega\left(G^{+}(0)\right)>0$, and we conclude from Theorem 7 that the plant decentralized integral controllable (DIC) with this choice of pairings.

\section{Notation}

$$
\begin{aligned}
& C(s)=\hat{C}(s) / s \text { - transfer function of decentralized (diagonal) con- } \\
& \text { troller with integral action } \\
& \hat{C}(s) \text {-transfer function of controller excluding integral } \\
& \text { action } \\
& G^{+}(0) \text { - plant steady-state gain matrix with the signs adjusted } \\
& \text { so that all diagonal elements have positive signs } \\
& G_{\text {diag }}=\operatorname{diag}\left\{g_{11}, g_{22}, \ldots, g_{n n}\right\} \text {-matrix consisting of the diagonal elements of the } \\
& \text { plant only } \\
& E=\left(G-G_{\text {diag }}\right) G_{\text {diag }}^{-1} \text {-interaction matrix (closely related to the Jacobi } \\
& \text { Iteration Matrix of Mijares et al. (1986)) } \\
& \lambda_{i}(A) \text {-eigenvalue } i \text { of the matrix } A \\
& \operatorname{Re}\{\lambda(A)\} \text {-real part of eigenvalues } \\
& \rho(A)=\max _{i}\left|\lambda_{i}(A)\right| \text {-spectral radius (magnitude of largest eigenvalue) } \\
& \mu(A) \text {-structured singular value (Doyle 1982) } \\
& |A| \text {-matrix } A \text { with all its elements replaced by its absolute } \\
& \text { value } \\
& \operatorname{RGA}(A)=A \times\left(A^{-1}\right)^{\mathrm{T}} \text { - Relative gain array of } A \text { (Bristol 1966) }
\end{aligned}
$$

\section{APPENDIX}

This appendix contains some basic mathematical terminology which may be useful for the reader. The following statements are all equivalent:

$A \Leftarrow B$; $A$ if $B$; $A$ is necessary for $B ; B \Rightarrow A$; only if $A$; $B$ is sufficient for $A$ if $B$ then $A$; and $\operatorname{not} A \Rightarrow$ not $B$

\section{REFERENCES}

BRISTOL, E. H. (1966). On a new measure of interaction for multivariable process control. IEEE Trans. on Automatic Control, 11, 133-134.

DoYLE, J. C. (1982). Analysis of feedback systems with structured uncertainty, IEE Proc. (UK), $129,242-250$.

ELAAHI, A. and LUYBEN, W. L. (1985). Control of an energy-conservative complex configuration of distillation column for four-component separations. Ind. Eng. Chem. Process Des. Dev., 24, 368-376.

Grosdidier, P., Morari, M. and Holt, B. R. (1985). Closed-loop properties from steady-state gain information. Ind. Eng. Chem. Fund., 24, 221-235.

GrosDIDIER, P. and MORARI, M. (1986). Interaction measures for systems under decentralized control. Automatica, 22, 309-319.

GrosDidIER, P. and MoraRI, M. (1987). A computer aided methodology for the design of decentralized controllers. Comput. chem. Engng., 11, 423-433.

Manousiouthakis, V., SAVAGE, R. and ARKUn, Y. (1986). Synthesis of decentralized controllers using the concept of block realative gain. AIChE Journal, 32, 991-1003.

McAvoy, T. J. (1983) InteractionnAnalysis-Principles and Applications (Instrument Society of America, Research Triangle Park, NC).

Mijares, G., Cole, J. D., Naugle, N. W., Preisig, H. A. and Holland, C. D. (1986). A new criterion for the pairing of control and manipulated variables. AIChE Journal, 32, 14391449. 
NETt, C. N. and SPANG, H. A. III (1987). Control structure design: a missing link in the evolution of modern control theory, presented at the 1987 American Control Conference, Minneapolis, June 1987.

NIEDERLINSKI, A. (1971). A heuristic approach to the design of linear multivariable control systems. Automatica, 7, 691.

Skogestad, S. and Morari, M. (1987). Letter to the Editor, AIChE Journal, 33, 701-702.

SKoGESTAD, S. and MORARI, M. (1989). Robust performance of decentralized control systems by independent designs. Automatica, 25, 119-125.

Yu, C.-C. and LuYBEN, W. L. (1986). Design of multiloop SISO controllers in multivariable processes. Ind. Eng. Chem. Process Des. Dev., bf 25, 498-503. 